\title{
The performance of rapid plasma reagin (RPR) titer in HIV-negative general paresis after neurosyphilis therapy
}

\author{
Ying Jiang ${ }^{1 * \dagger}$, Ruihui Weng ${ }^{1 \dagger}$, Yuefeng Zhang ${ }^{2 \dagger}$, Rong Fan ${ }^{1}$, Yulun Liu $^{3}$, Zhigang Chen ${ }^{1}$, Fuhua Peng ${ }^{1}$,
} Yong Chen ${ }^{3}$ and Xiaohong Chen ${ }^{*}$

\begin{abstract}
Background: Repeated nontreponemal serologic test for syphilis titers is recommended to evaluate treatment response. However, it is unknown whether serum rapid plasma reagin (RPR) titer can serve as a surrogate for determining the efficacy of treatment in general paresis (GP) remains unknown.

Methods: We retrospectively reviewed data from 105 GP patients, who were divided into two groups (62 CSF RPR+ patients and 43 CSF RPR- patients) according to reactive RPR test status in CSF. Clinical assessment included the Mini-Mental State Examination (MMSE) scores, CSF examinations (WBC count, protein concentration and RPR titer), and serum tests (RPR titer and TPPA). Among the 105 GP patients, 13 CSF RPR+ patients and 6 CSF RPR- patients had a 12 months follow-up of CSF, serum measures and MMSE.

Results: The median serum RPR titer was significantly higher in CSF RPR+ patients than that in CSF RPR- GP patients, 1: 8 [IQR 1:4-1:32] Vs. 1:4 [IQR 1:4-1:8] $(P<0.001)$. The number of CSF RPR+ patients with serum RPR titer $\geq 1: 32$ was significantly higher when compared with CSF RPR- patients $(P=0.001)$. For CSF RPR+ patients, the MMSE scores improved or remained constantly after penicillin treatment. For CSF RPR+ patients, the CSF RPR titer declined four-fold in $85 \%$ (11/13) of the patients, whereas the serum RPR titer declined four-fold in only $46 \%$ $(6 / 13)$ of the patients, the odds ratio is 6.4 (95\% confidence interval 1.0-41.2).

Conclusions: A four-fold decline in CSF RPR titer is a good predictor for treatment efficacy in CSF RPR+ GP patients within 12 months after the completion of therapy.
\end{abstract}

Keywords: Neurosyphilis, General paresis, Rapid plasma regain, HIV-negative

\section{Background}

Syphilis, which is caused by Treponema pallidum, is a major health problem worldwide. Cases of syphilitic dementia (general paresis, GP), which is characterized by rapidly progressive cognitive decline with or without psychiatric features, continue to be reported in the modern era [1].

Serum treponemal and non-treponemal tests are the most widely used methods for syphilis diagnosis. Reactive serum treponemal test (TT, including T. pallidum

\footnotetext{
* Correspondence: jiangying722@163.com; xiaohongchenzssy@aliyun.com ${ }^{\dagger}$ Equal contributors

${ }^{1}$ Department of Neurology, The Third Affiliated Hospital, Sun Yat-sen University, 600 Tianhe Road, Guangzhou, Guangdong 510630, People's Republic of China

Full list of author information is available at the end of the article
}

Haemagglutination test (TPHA) and T. pallidum Particle Agglutination test (TPPA)), symptoms and signs of neurosyphilis and reactive venereal disease research laboratory (VDRL) in cerebrospinal fluid (CSF) or CSF white blood cells $(\mathrm{WBCs})>5 / \mu \mathrm{l}$ or CSF protein $>45 \mathrm{mg} / \mathrm{dL}$ are used in the diagnosis of symptomatic neurosyphilis [2]. A reactive rapid plasma reagin (RPR) test in CSF is considered to be the diagnostic of neurosyphilis [2,3], but this test may be nonreactive in patients with GP depending on the criteria used to define neurosyphilis.

Repeated titers of nontreponemal (i.e., RPR, VDRL) serologic test for syphilis has been recommended to evaluate treatment response, with a four-fold decrease from baseline and/or seroreversion in 12-24 months after treatment representing an appropriate response to therapy/serologic 
cure [4]. For those neurosyphilis with symptoms or signs, resolution of clinical abnormalities is also considered to determine the efficacy of treatment [5].

The goal of this study is to explore whether serum RPR titer could serve as a surrogate for determining the efficacy of treatment in GP.

\section{Methods}

\section{Patients}

We retrospectively reviewed 105 GP patients (91 males and 14 females). These GP patients were late syphilis and fulfilled all of the following criteria: (1) TPPA and RPR titer were tested in serum and CSF, and were positive in serum TPPA; (2) serologic test was based on RPR positivity in CSF, or WBC count of CSF $>5$ cells $/ \mu \mathrm{L}$ or protein of CSF $>45 \mathrm{mg} / \mathrm{dL}$ with TPPA positivity in CSF [2]; (3) patient has slowly progressive deterioration in memory, personality, and habits, and with disorientation; (4) exclusion of other diagnosis. These patients were divided into two groups (62 CSF RPR+ patients and 43 CSF RPR- patients) according to reactive RPR status in CSF. Furthermore, since some GP patients were uncooperative, certain information was obtained from their relatives at admission. All patients involved in this study were diagnosed with GP for the first time and they have not received the recommended penicillin therapy [penicillin 18-24 million units intra-venous (IV) daily for 1014 days] before the diagnosis of GP. Cognitive assessment, the Chinese version of the Mini-Mental State Examination (MMSE), was tested in all cases. It covers the following five areas of cognition: orientation, memory, attention and calculation, recall and language [6]. WBC count, protein concentration and RPR titer in CSF, RPR titer and TPPA in serum were assessed. Human immunodeficiency virus (HIV) infection was excluded in all patients using serological testing.

Thirteen CSF RPR+ GP patients returned for follow-up visits with lumbar puncture, MMSE scores and blood samples in 12 months after penicillin treatment (penicillin G, 24 million units per day for 14 days) (see Additional file 1: Table S1). Fourteen CSF RPR- GP patients returned for follow-up visits with MMSE scores and serological tests in 12 months after penicillin treatment, while 6 of them had a follow-up evaluation of CSF (see Additional file 2: Table S2). An appropriate response to the therapy in the follow-up visit was defined as a four-fold decline in RPR titer which means four-fold decrease or returning to nonreactive in 12 months after treatment.

\section{Statistical analysis}

All statistical analyses were performed by the Statistical Program for Social Sciences (SPSS) software (version 22. 0 , Chicago, IL, USA). Associations between categorical variables were assessed using the $\chi^{2}$ test or Fisher's exact test. Associations between continuous variables and categorical variables were assessed using the Mann-Whitney $U$ test. $P<0.05$ was considered to be statistically significant.

\section{Ethics}

The study was conducted according to the principles expressed in the Declaration of Helsinki and approved by the Medical Ethics Committee of the Third Affiliated Hospital of Sun Yat-sen University. The patients or the guardians of patients with severe cognitive impairment provided written informed consent. Lumbar puncture was also performed with informed consent.

\section{Results}

Patients

One hundred and five GP patients were divided into two groups (62 CSF RPR+ patients and 43 CSF RPR-patients) according to reactive RPR test status in CSF. The characteristics and CSF abnormalities of GP patients with CSF RPR+ and CSF RPR- are shown in Tables 1 and 2. The serum RPR titers of CSF RPR+ and CSF RPR- patients are shown in Fig. 1. The median serum RPR titer was significantly higher in CSF RPR+ patients than that in CSF RPR- GP patients, $1: 8$ [IQR 1:4-1:32] vs. 1:4 [IQR 1:4-1:8] $(P<0.001$, see Table 1). The number of CSF RPR+ patients with serum RPR titer $\geq 1: 32$ was significantly higher when compared with CSF RPR- patients $(P=0.001)$. The number of CSF RPR + GP patients who had no abnormalities or only one abnormality on initial lumbar puncture was significantly different when compared with CSF RPR- patients (see Table 2). Furthermore, no significant correlation was found between the 1/CSF RPR titer and 1/serum RPR titer in CSF RPR+ GP patients. $(r=0.149, P=0.249)$.

\section{Changes of MMSE and RPR titer in serum and CSF}

As shown in Tables 3, 13 CSF RPR+ GP patients (including 12 males and 1 female) who received IV penicillin treatment (penicillin G, 24 million units per day for 14 days) had a follow-up evaluation including MMSE and RPR tests in both serum and CSF. The median MMSE change (the changes in MMSE score between last follow-up evaluation in 12 months after penicillin treatment and initial admission) was 4 (IQR 2-11). None of the patients who received the follow-up evaluation showed obvious cognitive deterioration on clinical examination. MMSE scores were higher at post-treatment as compared to pre-treatment $(P<0.001$, see Table 3). For CSF RPR+ patients, the CSF RPR titer declined four-fold in $85 \%(11 / 13)$ of the patients, whereas the serum RPR titer declined four-fold in only $46 \%(6 / 13)$ of the patients. The odds ratio is 6.4 (95\% confidence interval 1.0-41.2). The changes of CSF WBC and protein measures in 12 patients by follow-up are shown in Table 3.

Fourteen CSF RPR- GP patients (including 13 males and 1 female) had a follow-up evaluation of serum RPR 
Table 1 Characteristics, CSF and serum measures in CSF RPR+ and CSF RPR- patients

\begin{tabular}{|c|c|c|c|c|}
\hline Characteristic & All Patients $(n=105)$ & CSF RPR+ Patients $(n=62)$ & CSF RPR- Patients $(n=43)$ & $P^{a}$ \\
\hline Male sex & 91/105(87\%) & $54 / 62(87 \%)$ & $37 / 43(86 \%)$ & 0.876 \\
\hline Age, median years (IQR) & $52(47-58)$ & $53(47-58)$ & $52(47-56)$ & 0.432 \\
\hline MMSE (IQR) & $14(12-17)$ & $14(12-17)$ & $14(11-17)$ & 0.982 \\
\hline \multicolumn{5}{|l|}{ CSF WBC count } \\
\hline$>5$ cells $/ \mu l$ & $26 / 89(29 \%)$ & $14 / 46(30 \%)^{b}$ & $12 / 43(28 \%)$ & 0.793 \\
\hline Median cells/ml (IQR) & $3(1-6)$ & $3(0.75-6)$ & $2(1-6)$ & 0.878 \\
\hline \multicolumn{5}{|l|}{ CSF protein concentration } \\
\hline$>45 \mathrm{mg} / \mathrm{dl}$ & $73 / 89(82 \%)$ & $34 / 46(74 \%)^{b}$ & $39 / 43(91 \%)$ & 0.039 \\
\hline Median mg/dl (IQR) & $62(48-75)$ & $62.5(45-75.25)$ & $61(50-75)$ & 0.577 \\
\hline Reactive CSF RPR & $62 / 105(59 \%)$ & $62 / 62(100 \%)$ & $0 / 43(0 \%)$ & \\
\hline Median CSF RPR titer (IQR) & - & $1: 2(1: 2-1: 4)$ & - & \\
\hline \multicolumn{5}{|l|}{ Serum RPR titer } \\
\hline$\geq 1: 32$ & 21/108(19\%) & 19/62(31\%) & $2 / 43(4.7 \%)$ & 0.001 \\
\hline 1: 16 & 16/108(15\%) & $8 / 62(13 \%)$ & $8 / 43(18.6 \%)$ & 0.424 \\
\hline 1: 1-1: 8 & 68/108(63\%) & $35 / 62(56 \%)$ & $33 / 43(76.7 \%)$ & 0.032 \\
\hline Median serum RPR titer (IQR) & $1: 8(1: 4-1: 16)$ & $1: 8(1: 4-1: 32)$ & $1: 4(1: 4-1: 8)$ & $<0.001$ \\
\hline Normalized by final follow-up visit & $14 / 27(52 \%)$ & $6 / 13(46 \%)^{c}$ & $8 / 14(57 \%)^{d}$ & 0.568 \\
\hline
\end{tabular}

Data are no. (\%) of patients, unless otherwise indicated. IQR interquartile range, $R P R$ rapid plasma regain, MMSE mini-mental state examination

${ }^{a}$ Associations between categorical variables were assessed using the $\times 2$ test or Fisher's exact test. Associations between continuous variables and categorical variables were assessed using the Mann-Whitney $U$ test

${ }^{b}$ Data are for 46 patients

'Data are for 13 patients

${ }^{\mathrm{d}}$ Data are for 14 patients

titer (see Table 1), while 6 of them had a follow-up evaluation of both CSF and serum RPR titer. The changes of MMSE scores and serum and CSF measures in these 6 patients by follow-up are shown in Table 4 .

\section{Discussion}

In this study, we explored the correlation between the RPR titer and GP. Serum RPR titer $\geq 1: 32$ has been described as a risk factor for neurosyphilis with HIV infection regardless of the stage of syphilis [7-9]. In this study, we also found that the number of CSF RPR+ patients with serum RPR titer $\geq 1: 32$ was significantly higher when compared with CSF RPR- patients.

Table 2 Cerebrospinal fluid abnormalities in CSF RPR+ and CSF RPR- patients

\begin{tabular}{lllll}
\hline Characteristic & $\begin{array}{l}\text { All } \\
\text { Patients } \\
(n=89)\end{array}$ & $\begin{array}{l}\text { CSF RPR+ } \\
\text { Patients } \\
(n=46)\end{array}$ & $\begin{array}{l}\text { CSF RPR- } \\
\text { Patients } \\
(n=43)\end{array}$ & $P$ \\
\hline No abnormalities & $8 / 89(9 \%)$ & $8 / 46(17 \%)$ & $0 / 43(0)$ & 0.004 \\
One abnormality & $63 / 89(71 \%)$ & $28 / 46(61 \%)$ & $35 / 43(81 \%)$ & 0.033 \\
Two abnormalities & $18 / 89(20 \%)$ & $10 / 46(22 \%)$ & $8 / 43(19 \%)$ & 0.713
\end{tabular}

No abnormalities, no changes of CSF WBC count $(>5$ cells/ $\mu$ l) and CSF protein concentration ( $>45 \mathrm{mg} / \mathrm{dll})$; One abnormality, change in CSF WBC count ( $>5$ cells/ $\mu \mathrm{ll}$ ) or CSF protein concentration (>45 mg/dl); Two abnormalities, changes in CSF WBC count ( $>5$ cells $/ \mu$ l) and CSF protein concentration ( $>45 \mathrm{mg} / \mathrm{dl}$ ); RPR, rapid plasma regain
When patients have symptomatic neurosyphilis, the success of therapy is assessed by normalization of CSF abnormalities, including pleocytosis, elevated protein concentration, or a reactive CSF VDRL test, and by resolution of symptoms and signs [5]. Repeated determination of serum RPR titer is recommended to evaluate treatment response, with a four-fold decrease from baseline and/or seroreversion in 12-24 months after treatment representing an appropriate response to therapy/serologic cure [4]. Marra et al. found that a four-fold decline in serologic RPR

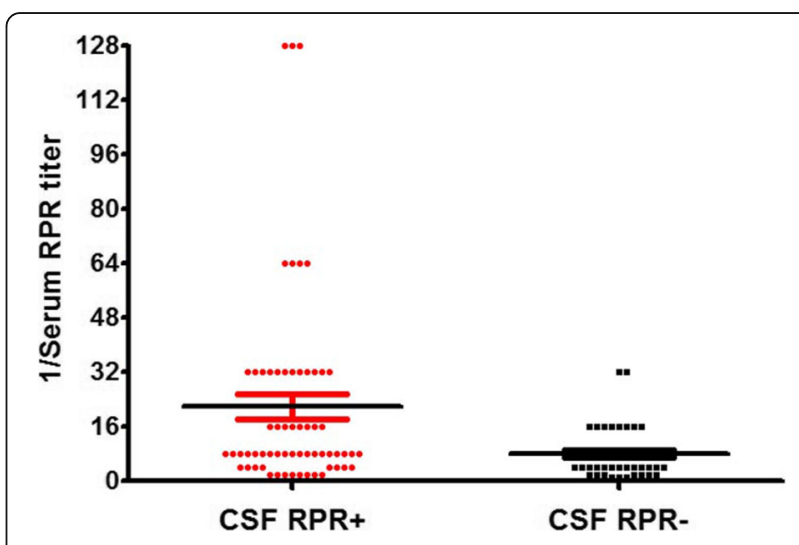

Fig. 1 The serum RPR titers for CSF RPR+ and CSF RPR- patients in a scatter plot 
Table 3 MMSE and changes of CSF measures and serum RPR titer in CSF RPR+ patients by follow-up

\begin{tabular}{|c|c|c|c|}
\hline Variable & $\begin{array}{l}\text { pre- } \\
\text { treatment }\end{array}$ & $\begin{array}{l}12 \text { months } \\
\text { after treatment }\end{array}$ & $P$ \\
\hline Increased CSF WBC count $^{a}$ & $5 / 12(42 \%)$ & $0 / 12(0 \%)$ & 0.012 \\
\hline $\begin{array}{l}\text { Increased CSF protein } \\
\text { concentration }^{\mathrm{a}}\end{array}$ & $7 / 12(58 \%)$ & $2 / 12(17 \%)$ & 0.035 \\
\hline Four-fold decline in CSF RPR & - & $11 / 13(85 \%)$ & - \\
\hline Four-fold decline in serum RPR & - & $6 / 13(46 \%)$ & - \\
\hline Median MMSE (IQR) & 13(9.5-15) & $19(16.5-20.5)$ & $<0.001$ \\
\hline
\end{tabular}

Increased CSF WBC count means CSF WBC count $>5$ cells $/ \mu$ l; Increased CSF protein concentration means CSF protein concentration $>45 \mathrm{mg} / \mathrm{dl}$; RPR, rapid plasma regain; MMSE, mini-mental state examination; ${ }^{a}$ Data are for 12 patients

titers correlated with resolution of CSF parameters in persons with neurosyphilis, and normalization of serum RPR titer correctly predicted treatment success of neurosyphilis [10]. However, we found a four-fold decline in CSF RPR titer is more likely to occur than four-fold decline in serum RPR titer among these CSF RPR+ GP patients after penicillin treatment. Reactive non-treponemal test in non-HIVinfected late (latent) syphilis patients often remains stable after adequate therapy [2]. Many studies have found the serological response of nontreponemal tests (ie, VDRL, RPR) to treatment was influenced by syphilis stage but not by HIV infection or reinfection [11-14]. Compared to primary syphilis, later stages of syphilis showed a significantly slower treatment response [11, 13-17]. In this study, all the GP patients were late syphilis, which probably explains the 4-fold decline in serum RPR titers might not adequately predict treatment efficacy in GP patients. The majority of patients were early neurosyphilis in the study by Marra et al. [10], which could partially explain why a four-fold decline in CSF RPR titer instead of the serum RPR normalization found by Marra is a good predictor of treatment efficacy in HIV-negative CSF RPR+ GP patients in our finding. According to these results, it should be emphasized that the disease stage of syphilis might lead to different treatment response. Different recommendations may

Table 4 MMSE and changes of CSF measures and serum RPR titer in CSF RPR- patients by follow-up

\begin{tabular}{llll}
\hline Variable & $\begin{array}{l}\text { pre- } \\
\text { treatment }\end{array}$ & $\begin{array}{l}12 \text { months } \\
\text { after treatment }\end{array}$ & $P$ \\
\hline Increased CSF WBC count & $2 / 6(33 \%)$ & $1 / 6(17 \%)$ & 0.505 \\
Increased CSF protein & $6 / 6(100 \%)$ & $1 / 6(17 \%)$ & 0.003 \\
concentration & & - & - \\
Four-fold decline in CSF RPR & - & $3 / 6(50 \%)$ & - \\
Four-fold decline in serum RPR & - & $17(13-19.25)$ & 0.048 \\
\hline Median MMSE (IQR) & $14.5(11-18.25)$ & 175 )
\end{tabular}

Increased CSF WBC count means CSF WBC count $>5$ cells/ $\mu$ l; Increased CSF protein concentration means CSF protein concentration $>45 \mathrm{mg} / \mathrm{dl}$; RPR, rapid plasma regain; MMSE, mini-mental state examination be needed for the follow-up of neurosyphilis depending on the stage of the disease.

During the follow-up at 12 months after penicillin treatment, no significant difference was found in the four-fold decline in serum RPR titer between CSF RPR+ and CSF RPR-patients. According to our data and our analyses, for CSF RPR- patients, normalization of CSF measures, not serum RPR titer value, is a sensible way of assessing treatment efficacy. A cutoff of greater than or equal to 5 cells/ $\mu \mathrm{L}$ has been the standard for determining normal CSF values. For CSF RPR- GP patients, CSF WBC count was often normal before treatment, so it is difficult to evaluate the treatment efficacy by assessing the recovery of CSF WBC. CSF protein concentration in neurosyphilis is slow to normalize and may remain elevated even after the normalization of other CSF and clinical abnormalities $[10,18]$. Thus, the decision to re-treat should not be based solely on failure of CSF protein concentration to normalize [1]. Importantly, our study found that the number of CSF RPR- patients (91\%) with CSF protein concentration $>45 \mathrm{mg} / \mathrm{dl}$ was significantly higher when compared with CSF RPR+ patients $(74 \%)(P=0.028)$. The normalization of CSF protein concentration occurred in most of CSF RPR- GP patients by follow-up, which suggested that it may be a good indicator of the success of therapy in GP, although the number of this patient group by follow-up is relatively small. Further studies should be conducted to provide more evidence on this question.

Some limitations of our study should be considered. Specifically, a) the study is a retrospective study, in which case the association may be confounded or modified by patient characteristics; b) the large percentage of loss of follow-up leads to small number of the patients with follow-up, which has limited the statistical power; c) further studies are required to assess an association between RPR titers and the clinical features of these cases, and to identify an ideal indicator of the success of therapy for CSF-RPR- GP patients.

\section{Conclusions}

The practical implications of our findings are that a four-fold decline in CSF RPR titer is a good predictor for treatment efficacy in CSF RPR + GP patients within 12 months after completion of therapy. The sole use of serum RPR titer might not adequately predict treatment efficacy of GP. Considering the limitations of this study, larger samples and more follow-up data are needed for future investigation. Furthermore, the association between RPR titers and the clinical features of these cases need to systematically quantified, and more indicators of treatment efficacy need to be investigated for CSF RPR- GP patients. 


\section{Additional files}

Additional file 1: Table S1. Follow-up of MMSE, CSF and serum measures in CSF-RPR+ patients. Thirteen CSF RPR+ GP patients returned for follow-up visits with lumbar puncture, MMSE scores and blood samples in 12 months after penicillin treatment. (DOCX $19 \mathrm{~kb})$

Additional file 2: Table S2. Follow-up of MMSE, CSF and serum measures in CSF-RPR- patients. Fourteen CSF RPR- GP patients returned for follow-up visits with MMSE scores and serological tests in 12 months after penicillin treatment, while 6 of them had a follow-up evaluation of CSF. (DOCX 19 kb)

\section{Abbreviations}

CSF: cerebrospinal fluid; GP: general paresis; MMSE: Mini-Mental State Examination; RPR: rapid plasma regain; TPPA: T. pallidum Particle Agglutination test; VDRL: Venereal disease research laboratory; WBC: white blood cells

\section{Acknowledgements}

The authors thank Dr. Christina Marra from University of Washington for helpful comments on this manuscript.

\section{Funding}

This work was supported by grants (to $X$ Chen) from the Israel Science Foundation (ISF)-the National Natural Science Foundation of China (NSFC) joint program (813111290) and by grant (to Y Jiang) from the Natural Science Foundation of Guangdong Province (2016A030313228) and the National Natural Science Foundation of China (81671182). Yong Chen and Yulun Liu's research were supported in part by National Institutes of Health under award numbers 1R01LM012607 and 1R01Al130460. The funders had no role in study design, data collection and analysis, decision to publish, or preparation of the manuscript.

\section{Availability of data and materials}

The datasets used and/or analysed during the current study are available from the corresponding author on reasonable request.

\section{Authors' contributions}

$Y J$ and XC contributed to the conception and design of the study. YJ, RW, $Y Z, R F, Y L, Z C, F P$ and $X C$ collected and organized the data. YJ, YL and YC analyzed the data. YJ and XC drafted the manuscript. All authors read and approved the final manuscript.

\section{Ethics approval and consent to participate}

The study was conducted according to the principles expressed in the Declaration of Helsinki and approved by the Medical Ethics Committee of the Third Affiliated Hospital of Sun Yat-sen University. The patients or the guardians of patients with severe cognitive impairment provided written informed consent. Lumbar puncture was also performed with informed consent.

\section{Consent for publication}

Not applicable.

\section{Competing interests}

The authors declare that they have no competing interest.

\section{Publisher's Note}

Springer Nature remains neutral with regard to jurisdictional claims in published maps and institutional affiliations.

\section{Author details}

'Department of Neurology, The Third Affiliated Hospital, Sun Yat-sen University, 600 Tianhe Road, Guangzhou, Guangdong 510630, People's Republic of China. 'Department of Neurology, Guangzhou Brain Hospital, Affiliated Brain Hospital of Guangzhou Medical University, 36 Mingxin Road, Guangzhou, Guangdong 510370, People's Republic of China. ${ }^{3}$ Department of Biostatistics, Epidemiology and Informatics, University of Pennsylvania, Philadelphia, PA 19104, USA.
Received: 25 June 2017 Accepted: 25 March 2018

Published online: 02 April 2018

\section{References}

1. Marra CM. Neurosyphilis. Continuum (Minneap Minn). 2015;21(6):1714-28.

2. French $P$, Gomberg $M$, Janier $M$, Schmidt $B$, van Voorst Vader $P$, Young $H$. IUST. IUSTI: 2008 European guidelines on the Management of Syphilis. Int J STD AIDS. 2009;20(5):300-9.

3. Castro R, Prieto ES, da Luz Martins Pereira F. Nontreponemal tests in the diagnosis of neurosyphilis: an evaluation of the venereal disease research laboratory (VDRL) and the rapid plasma Reagin (RPR) tests. J Clin Lab Anal. 2008;22(4):257-61

4. Workowski KA, Bolan GA. Centers for Disease Control and Prevention. Sexually transmitted diseases treatment guidelines, 2015. MMWR Recomm Rep. 2015:64(RR-03):1-137.

5. Chahine LM, Khoriaty RN, Tomford WJ, Hussain MS. The changing face of neurosyphilis. Int J Stroke. 2011;6(2):136-43.

6. Su X, Shang L, Xu Q, Li N, Chen J, Zhang L, Zhang L, Hua Q. Prevalence and predictors of mild cognitive impairment in Xi'an: a community-based study among the elders. PLoS One. 2014;9:e83217.

7. Marra CM, Maxwell CL, Smith SL, Lukehart SA, Rompalo AM, Eaton M, Stoner BP, Augenbraun M, Barker DE, Corbett JJ, Zajackowski M, Raines C, Nerad J, Kee R, Barnett SH. Cerebrospinal fluid abnormalities in patients with syphilis: association with clinical and laboratory features. J Infect Dis. 2004;189(3): 369-76.

8. Libois A, De Wit S, Poll B, Garcia F, Florence E, Del Rio A, Sanchez P, Negredo E, Vandenbruaene M, Gatell JM, Clumeck N. HIV and syphilis: when to perform a lumbar puncture. Sex Transm Dis. 2007;34(3):141-4.

9. Ghanem KG, Moore RD, Rompalo AM, Erbelding EJ, Zenilman JM, Gebo KA. Neurosyphilis in a clinical cohort of HIV-1-infected patients. AIDS. 2008:22(10):1145-51.

10. Marra CM, Maxwell CL, Tantalo LC, Sahi SK, Lukehart SA. Normalization of serum rapid plasma reagin titer predicts normalization of cerebrospinal fluid and clinical abnormalities after treatment of neurosyphilis. Clin Infect Dis. 2008:47(7):893-9.

11. Knaute DF, Graf N, Lautenschlager S, Weber R, Bosshard PP. Serological response to treatment of syphilis according to disease stage and HIV status. Clin Infect Dis. 2012:55(12):1615-22.

12. Hutchinson CM, Hook E 3rd, Shepherd M, Verley J, Rompalo AM. Altered clinical presentation of early syphilis in patients with human immunodeficiency virus infection. Ann Intern Med. 1994;121:94-100.

13. Gourevitch MN, Selwyn PA, Davenny K, Buono D, Schoenbaum EE, Klein RS, Friedland $\mathrm{GH}$. Effects of HIV infection on the serologic manifestations and response to treatment of syphilis in intravenous drug users. Ann Intern Med. 1993;118(5):350.

14. Riedner G, Rusizoka M, Todd J, Maboko L, Hoelscher M, Mmbando D, Samky E, Lyamuya E, Mabey D, Grosskurth H, Hayes R. Single-dose azithromycin versus penicillin $\mathrm{G}$ benzathine for the treatment of early syphilis. N Engl J Med. 2005;353:1236-44.

15. Romanowski B, Sutherland R, Fick GH, Mooney D, Love EJ. Serologic response to treatment of infectious syphilis. Ann Intern Med. 1991;114: 1005-9.

16. Ghanem KG, Erbelding EJ, Wiener ZS, Rompalo AM. Serological response to syphilis treatment in HIV-positive and HIV-negative patients attending sexually transmitted diseases clinics. Sex Transm Infect. 2007:83:97-101.

17. Gonzalez-Lopez JJ, Guerrero ML, Lujan R, Tostado SF, de Gorgolas M, Requena $\mathrm{L}$. Factors determining serologic response to treatment in patients with syphilis. Clin Infect Dis. 2009;49:1505-11.

18. Marra CM, Maxwell CL, Tantalo L, Eaton M, Rompalo AM, Raines C, Stoner BP, Corbett JJ, Augenbraun M, Zajackowski M, Kee R, Lukehart SA. Normalization of cerebrospinal fluid abnormalities after neurosyphilis therapy: does HIV status matter? Clin Infect Dis. 2004;38(7):1001-6. 